International Mathematical Forum, 2, 2007, no. 39, 1935 - 1943

\title{
A New Method for Finding Solution of Nonhomogeneous Difference Equations
}

\author{
Hassan Hosseinzadeh and G. A. Afrouzi ${ }^{1}$ \\ Islamic Azad University, Ghaemshahr Branch \\ P.O. Box 163, Ghaemshahr, Iran
}

\begin{abstract}
In this paper we introduce the shift operator $E$ and express some of it's properties, particularly shift exponential formula is as follows:

$$
P(E)\left(r^{n} f_{n}\right)=r^{n} P(r E) f_{n},
$$

where $P$ is a polynomial with constant coefficients. In the sequel we solve the difference equation " $(E-r) k y n=f n$ ", firstly, and expand the result of finding solution of nonhomogeneous difference equations with constant coefficients where these equations written in the following form:

$$
P(E) y_{n}=f_{n},
$$

where $y$ is defined in the closed interval $I=[a, b]$.

Keywords: Shift operator; Shift exponential formula; Difference equation;
\end{abstract} Particular solution

\section{Preliminaries}

1.1 Shift operator $E$ " $E y_{n}=y_{n+1}$ "is a linear operator, that is one of the most important tools in Numerical Analysis, used in Interpolations and Numerical solution of ODE and PDE. This operator is used as fundamental element in the structure of Difference equations. The Difference equations appear in Numerical solution of ordinary differential equations, Partial differential equation, Integral equations, Integro- differential equation, [1], [2], [3], [4], [5]. Nonlinear difference equation (Euler method, Euler Modified method, Midpoint method,) appears mostly in numerical solution of initial value problem

\footnotetext{
${ }^{1}$ Corresponding author E-mail: afrouzi@umz.ac.ir also both authors are in : Department of Mathematics, Faculty of Basic Science, Mazandaran University, Babolsar, Iran.
} 
$" y^{\prime}=f(x, y), y\left(x_{0}\right)=y_{0} "$ but the linear difference equation appears in numerical solution of initial condition problems or boundary value problems [2], [3].

Definition1. Let $y_{0}, y_{1}, y_{2}, \ldots, y_{m}$ be the values of function $y(n)$ in the nodes of interval $I=[a, b]=\left[x_{0}, x_{m}\right]$ such that $x_{j+1}-x_{j}=h, j=0,1, \ldots, m-1$, then the following equation is said difference equation of order $n$.

$$
F\left(j, y_{j}, y_{j+1}, \ldots, y_{j+n}\right)=0 .
$$

Difference equations are of two types, we discuss the linear difference equation with constant coefficients, these equations can be written by the shift operator $E$ as follows:

$$
\left(\sum_{j=0}^{m} a_{j} E^{j}\right) y_{n}=P(E) y_{n}=f_{n},
$$

where $P$ is a polynomial with degreem, $f_{n}$ is a real- valued function of $n$.

(i) if $f_{n}=0$, then (2) is called homogeneous.

(ii) if $f_{n} \neq 0$, then (2) is called non-homogeneous.

In this paper we try to find the particular solution of nonhomogeneous difference equations.

\subsection{Some properties of the shift operator $E$}

$$
\begin{gathered}
(E-a) a^{n}=0, \quad n=0,1,2,3, \ldots \\
(E-a)^{m}\left(n^{m} a^{n}\right)=m ! a^{m+n}, \quad m=0,1,2,3, \ldots \\
(E-a)^{m+j}\left(n^{m} a^{n}\right)=m ! a^{m+n}, \quad m=0,1,2,3, \ldots, \quad j=0,1,2,3, \ldots
\end{gathered}
$$

Remark 1. Identity (3) is proved by the Mathematical induction.

\subsection{Some properties of polynomial $P(E)$ and solution of difference equations.}

Suppose that $r$ is not zero of $P$ i.e. $P(r) \neq 0$, then

$$
P(E) r^{n}=P(r) r^{n}
$$


If $r$ is the iterated root of order $k$ for polynomial $P$, that is

$$
P(r)=P^{\prime}(r)=\ldots=P^{k-1}(r)=0, P^{k}(r) \neq 0
$$

then

$$
P_{m}^{(k)}(r)=k ! P_{m-k}(r)
$$

where $P_{m}(E)=(E-r)^{k} P_{m-k}(E)$ and $P_{m}^{k}(E)=k ! P_{m-k}(E)+o(E-r)$ we obtain.

$$
\begin{gathered}
P_{m}^{(k)}(E)\left(n^{k} r^{n}\right)=k ! P_{m-k}(E)\left(n^{k} r^{n}\right) . \\
P_{m}(E)\left(n^{k} r^{n}\right)=P_{m-k}(E)(E-r)\left(n^{k} r^{n}\right)=k ! P^{(k)}(r) r^{n+k} .,
\end{gathered}
$$

Inversion operator of identities (4), (5), (7) and (8) will be written as follow respectively

$$
\frac{1}{(E-r)^{k}} r^{n}=\frac{1}{k !} n^{k} r^{n-k}
$$

For example, $\frac{1}{(E-2)^{5}} 2^{n}=\frac{1}{5 !} n^{5} 2^{n-5}$.

$$
\begin{gathered}
\frac{1}{P(E)} r^{n}=\frac{1}{P(r)} r^{n}, \quad P(r) \neq 0 ., \\
\frac{1}{P(E)} r^{n}=\frac{1}{k ! P^{(k)}(r)}\left(n^{k} r^{n-k}\right), \\
P(r)=P^{\prime}(r)=\ldots=P(r)^{(k-1)}=0, p^{(k)}(r) \neq 0 .
\end{gathered}
$$

for instance, consider $\frac{1}{E^{4}-8 E^{2}+16} r^{n}=\frac{1}{2 ! \times 32} 2^{n-3} n^{2}=2^{n-9} n^{2}$ and $\frac{1}{E^{4}-8 E^{2}+16}(-2)^{n}=\frac{1}{2 ! \times(-32)}(-2)^{n-3} n^{2}=-(-2)^{n-9} n^{2}$

Theorem 1(shift exponential).Let $P$ be a polynomial with degree $k$, then 


$$
P_{m}\left(r^{n} y_{n}\right)=r^{n} P(r E) y_{n}
$$

Proof. By considering

$$
\begin{aligned}
P(E)\left(r^{n} y_{n}\right) & =\left(\sum_{i=0}^{k} a_{i} E^{i}\right)\left(r^{n} y_{n}\right)=\sum_{i=0}^{k} a_{i} r^{i+n} y_{i+n} \\
& =r^{n}\left(\sum_{i=0}^{k} a_{i}(r E)^{i}\right) y^{n}=r^{n} P(r E) y_{n}
\end{aligned}
$$

The proof is easy.

Remark 2. Under the inversion operator, Identity (12) can be written as follows:

$$
P_{m}\left(r^{n} y_{n}\right)=r^{n} P(r E) y_{n}
$$

Definition 2. Each of both formulas (12) and (13) are called shift exponential formula. These formulas are used for finding particular solution of non-homogeneous difference equations with constant coefficients in the form of $\left(\sum_{i=0}^{k} a_{i} E^{i}\right) y_{n}=f_{n}$

Particular type 1. Let $P(E)=(E-r)^{k}$ then

$$
P(E)\left(r^{n} y_{n}\right)=(E-r)^{k}\left(r^{n} y_{n}\right)=r^{n+k}(E-1)^{k} y_{n}
$$

Particular type 2. Let $y_{n}=n^{m}(14)$ becomes

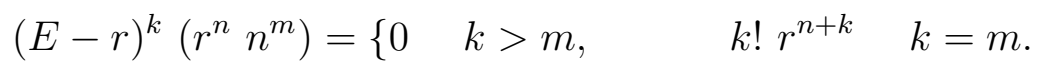

These results are used in finding solution of non-homogeneous difference equations with constant coefficients. Under the inversion operators in formulas (14) and (15) we have

$$
\begin{gathered}
\frac{1}{(E-r)^{k}}\left(r^{n} y_{n}\right)=r^{n-k} \frac{1}{(E-1)^{k}} y_{n} . \\
\frac{1}{(E-r)^{k}}\left(r^{n}\right)=\frac{1}{k !} n^{k} r^{n-k} .
\end{gathered}
$$

\section{Example .}




$$
\frac{1}{(E-2)^{3}}\left(2^{n+4}\left(3 n^{2}+6 n+5\right)\right)=2^{n+1} \frac{1}{(E-1)^{3}}\left(3 n^{2}+6 n+5\right)=\frac{1}{15} 2^{n} n^{5}
$$

2.4. Effect of operator $E-r$ on exponential function $a^{n}(a \neq r)$ $(E-r) a^{n}=a^{n+1}-r a^{n}=(a-r) a^{n}$ it implies

$$
\frac{1}{(E-r)}=\frac{a^{n}}{a-r}
$$

Generally

$$
(E-r)^{k} a^{n}=(a-r)^{k} a^{n} .
$$

Identity (19) is proved by the mathematical induction, under the inversion operator, identity (19) becomes

$$
\frac{1}{(E-r)^{k}} a^{n}=\frac{a^{n}}{(a-r)^{k}}
$$

for example, $\frac{1}{(E-2)^{4}}\left(3^{n}\right)=\frac{3^{n}}{(3-2)^{4}}=3^{n}$.

Particular case. Let $r=1$, then (19) and (20) can be written as follows, respectively

$$
\frac{1}{(E-1)} a^{n}=\frac{a^{n}}{(a-1)}, \quad \frac{1}{(E-1)^{k}} a^{n}=\frac{a^{n}}{(a-1)^{k}} .
$$

Theorem 2. Prove that

$$
\frac{1}{(E-r)} f_{n}=\sum_{j=0}^{n-1} r^{n-j-1} f_{j}
$$

Proof. Is easy, consider

$$
(E-r) \sum_{j=0}^{n} r^{n-j-1} f_{j}=\sum_{j=0}^{n+1} r^{n-j} f_{j}-r \sum_{j=0}^{n} r^{n-j-1} f_{j}=f_{n} .
$$

Therefore $\frac{1}{(E-r)} f_{n}=\sum_{j=0}^{n-1} r^{n-j-1} f_{j}$.

Identity (21) is used in solution of non-homogeneous difference equations with constant coefficients.

Particular case. Suppose that $r=1$, then (21) becomes

$$
\frac{1}{(E-1)} f_{n}=\sum_{j=0}^{n-1} f_{j}
$$


Example 1.

$$
\frac{1}{(E-r)} n^{3}=\sum_{j=0}^{n-1} j^{3}=\left(\sum_{j=0}^{n-1} j\right)^{2}=\frac{n^{2}(n-1)^{2}}{4}
$$

Example 2.

$$
\begin{gathered}
\frac{1}{(E-2)}\left(2^{n} n^{3}\right)=2^{n-1} \frac{1}{E-1} n^{3}=2^{n-1} \sum_{j=1}^{n-1} j^{3} \\
=2^{n-1} \times \frac{n^{2}(n-1)^{2}}{4}=2^{n-3} n^{2}(n-1)^{2} .
\end{gathered}
$$

Example 3. Evaluate the particular solution of the difference equation

$$
(E-2) y_{n}=\ln \left(\frac{2 n^{2}+4 n+13}{n^{2}+2 n+10} .\right.
$$

Solution. Using the formula(22) we have

$$
y_{n p}=\frac{1}{(E-1)} \ln \left(\frac{2 n^{2}+4 n+13}{n^{2}+2 n+10}=\ln \left(n^{2}+2 n+10\right) .\right.
$$

Example 4. Find the particular solution of the following difference equation

$$
(E-3) y_{n}=3^{n+1} \cos \frac{n \pi}{2} .
$$

Solution. Using the formulas(13) and (21), we have

$$
y_{n p}=\frac{1}{(E-3)}\left(3^{n+1} \cos \frac{n \pi}{2}\right)=\frac{3^{n}}{(E-1)}\left(\cos \frac{n \pi}{2}\right)=\frac{3^{n}}{2}\left(\sin \frac{n \pi}{2}-\cos \frac{n \pi}{2}\right)
$$

\subsection{Solution of non-homogeneous difference equations with constant coefficients.}

Consider: $P(E) y_{n}=f_{n}$, where $P$ is a polynomial of degree $m$, we can write the particular solution of it as follows

$$
y_{n p}=\frac{1}{P(E)} f_{n}
$$

(i) Suppose that $P$ has the $m$ distinct real zeros $\alpha_{1}, \alpha_{2}, \ldots, \alpha_{m}$ then the decomposition of fraction $\frac{1}{P(E)}$ can be written as $\frac{1}{P(E)}=\sum_{j=1}^{m} \frac{A_{j}}{E-\alpha_{j}}$ where $\lim _{r \rightarrow \alpha_{j}} \frac{r-\alpha_{j}}{P(r)}, j=$ $1,2, \ldots, m$. 
Consequently, the particular solution can be written as follows

$$
y_{n p}=\left(\sum_{j=1}^{m} \frac{A_{j}}{E-\alpha_{j}}\right) f_{n}=\sum_{j=1}^{m}\left(A_{j} \frac{1}{E-\alpha_{j}} f_{n}\right) .
$$

(ii) if many zeros of $P$ are complex numbers, in this case we will use identity (23) for finding the particular solution, too.

Remark 3. For finding particular solution we will use the iterated divisions as follows $y_{n p}=\frac{1}{E-\alpha_{m}}\left(\frac{1}{E-\alpha_{m-1}}\left(\ldots \frac{1}{E-\alpha_{1}}\left(f_{n}\right) \ldots\right)\right)$.

Remark 4. It is not possible decomposition for iterated zero, namely (E$r)^{k} y_{n}=f_{n}, y_{n p}=\frac{1}{(E-r)^{k}} f_{n}$ thus it is possible iterative divisions only as $y_{n p}=\frac{1}{E-r}\left(\frac{1}{E-r}\left(\ldots \frac{1}{E-r}\left(f_{n}\right) \ldots\right)\right)$.

Example 1. Find the particular solution of the following difference equation:

$$
\left(36 E^{3}-36 E^{2}+11 E-1\right) y_{n}=6^{-n}\left(6 n^{2}-12 n-10\right) .
$$

Solution. We have

$$
\begin{aligned}
y_{n p} & =\frac{1}{36 E^{3}-36 E^{2}+11 E-1}\left(6^{-n}\left(6 n^{2}-12 n-10\right)\right) \\
& =6^{-n+1} \frac{1}{(E-1)(E-2)(E-3)}\left(6 n^{2}-12 n-10\right) \\
& =6^{-n+1}\left(\frac{2}{E-1}-\frac{1}{E-2}-\frac{\frac{1}{2}}{E-3}\right)\left(6 n^{2}-12 n-10\right) \\
& =6^{-n+1} n^{3} .
\end{aligned}
$$

Example 2. Find the particular solution of the following difference equation of order four: $\left(E^{4}+4 E^{2}+16\right) y_{n}=2^{n} \sin \frac{n \pi}{3}$.

Solution. By division operation, write

$$
\begin{gathered}
y_{n p}=\frac{1}{E^{4}+4 E^{2}+16}\left(2^{n} \sin \frac{n \pi}{3}\right)=2^{n} \frac{1}{16 E^{4}+16 E^{2}+16}\left(\sin \frac{n \pi}{3}\right) \\
2^{n-4} E^{4}+E^{2}+1\left(\sin \frac{n \pi}{3}\right)=2^{n-5}\left(\frac{E}{E^{2}-E+1}-\frac{E}{E^{2}+E+1}\left(\sin \frac{n \pi}{3}\right)\right. \\
2^{n-5}\left(\frac{1}{E^{2}-E+1}-\frac{1}{E^{2}+E+1}\left(\sin (n+1) \frac{\pi}{3}=2^{n-5}\left(\cos \frac{n \pi}{3}+2 \sin \frac{n \pi}{3}\right) .\right.\right.
\end{gathered}
$$


Example 3. Find the particular solution of the difference equation as follows

$$
\left(E^{3}+6 E^{2}+12 E-8\right) y_{n}=2^{n} \sin \frac{n \pi}{2} .
$$

Solution. We will write

$$
\begin{aligned}
& y_{n p}=\frac{1}{(E-2)^{3}}\left(2^{n} \sin \frac{n \pi}{2}\right)=2^{n-3} \frac{1}{(E-1)^{3}}\left(\sin \frac{n \pi}{2}\right) \\
& \left.=2^{n-3} \times \frac{1}{4}\left(\sin \frac{n \pi}{2}-\cos \frac{n \pi}{2}\right)=2^{n-5} \sin \frac{n \pi}{2}-\cos \frac{n \pi}{2}\right) .
\end{aligned}
$$

Corollary 1. By substituting $y_{n}=Y_{n} r^{n}$ in the difference equation $(E-$ $r)^{k} y_{n}=r^{n+k} f_{n}$, we will obtain the difference equation

$$
(E-1)^{k} y_{n}=f_{n}
$$

Corollary 2. Setting $y_{n}=a^{n} Y_{n}$ in the difference equation " $P(E) y_{n}=a^{n} f_{n} "$ , we will obtain the difference equation " $P(a E) y_{n}=f_{n}$ " where can be in one of the following forms:

$A \sin \alpha n+B \cos \alpha n, Q_{k}(n)$ (polynomial of degree k of variable n) and $Q_{k}(n)(A \sin \alpha n+$ $B \cos \alpha n)$.

Example 1. Solve the following difference equation and find it's particular solution $(E-2)^{2} y_{n}=\left(2^{n+3}+2^{n}\right) n$.

Solution. Substituting $y_{n}=2^{n} Y_{n}$, It is $(E-1) Y_{n}=6 n \quad, \quad y_{n p}=$ $\frac{1}{(E-1)^{2}}(6 n)=n^{2}(n-3)$,

consequently: $y_{n p}=2^{n} Y_{n p}=2^{n} n^{2}(n-3)$.

Example 2. Calculate the particular solution of the following difference equation

$$
(E-4)^{3} y_{n}=2^{2 n+9}(3 n+6) .
$$

Solution. Setting $y_{n}=4^{n} Y_{n}$ we obtain

$$
\begin{gathered}
(E-1)^{3} Y_{n}=24 n+48, \quad y_{n}=\frac{1}{(E-1)^{3}}(24 n+48) \\
Y n p=\frac{1}{2}(-n+22) n^{3},
\end{gathered}
$$

using (21) three times yields.

consequently $y_{n p}=Y_{n p}=2^{2 n-1}(-n+22) n^{3}$. 


\section{Discussion and results}

The shift operator E method in solving of non-homogeneous difference equations with constant coefficients is a new method which we can solve all of NHDE with constant coefficients by using this method.

\section{References}

[1] Hildel brand, F.B., "Introduction to Numerical Analysis", Mc. GrawHill, New.York, 1956.

[2] Isaacson, E. and H.B., "Analysis of Numerical Methods", John Wiley and Sons,..New York, 1966.

[3] Lambert, J.D. "Computation methods in ordinary Differential Equations" John .Wiley and Sons, New York, 1973.

[4] Phillips, G.M., Taylor P.J. "Theory and Applications of Numberical Analysis", Fifth. Edition, Academic press, 1980.

[5] Ralston, A. "A first course in Numerical Analysis "Mc. Graw-Hill, New York.

Received: November 5, 2006 